\title{
Computational Modeling of Threat Learning Reveals Links with Anxiety and Neuroanatomy
}

Rany Abend ${ }^{1}$, PhD; Sonia G. Ruiz ${ }^{1}$, BA; Andrea L. Gold ${ }^{1,2}$, PhD; Julia L. Napoli ${ }^{6}$, BS; Jennifer C. Britton ${ }^{1,3}$, PhD; Kalina J. Michalska ${ }^{1,4}, \mathrm{PhD}$; Tomer Shechner ${ }^{1,5}, \mathrm{PhD}$; Anderson M. Winkler ${ }^{1}$, MD; Ellen Leibenluft ${ }^{1}$, MD; Daniel S. Pine ${ }^{1}, \mathrm{MD}$; Bruno B. Averbeck ${ }^{6}, \mathrm{PhD}$

${ }^{1}$ Emotion and Development Branch, National Institute of Mental Health, National Institutes of Health, Bethesda, MD

${ }^{2}$ Department of Psychiatry and Human Behavior, Brown University Warren Alpert Medical School, Providence, RI

${ }^{3}$ Department of Psychology, University of Miami, Coral Gables, FL

${ }^{4}$ Department of Psychology, University of California Riverside, Riverside, CA

${ }^{5}$ Psychology Department, University of Haifa, Haifa, Israel

${ }^{6}$ Laboratory of Neuropsychology, National Institute of Mental Health, National Institutes of Health, Bethesda, MD

Corresponding author: Rany Abend, PhD. Address: Emotion and Development Branch, National Institute of Mental Health, 9000 Rockville Pike, Bldg 15K, Bethesda, Maryland 20892. Phone: (301) 594-9144. Fax: (301) 594-9959. Email: rany.abend@nih.gov. 


\begin{abstract}
Influential theories implicate variations in the mechanisms supporting threat learning in variations in the severity of anxiety symptoms. We use computational models of associative learning in conjunction with structural imaging to explicate links among the computational mechanisms underlying threat learning, their neuroanatomical substrates, and anxiety severity. We recorded skin-conductance data during a threat-learning task from individuals with and without anxiety disorders ( $\mathrm{N}=251 ; 8-50$ years; 116 females). Reinforcement-learning model variants quantified processes hypothesized to relate to anxiety: threat conditioning, threat generalization, safety learning, and threat extinction. We then tested associations among latent learning parameters, whole-brain morphometry, and anxiety severity, as well as age moderation. Results indicate that greater anxiety severity related specifically to slower safety learning and threat extinction. Gray-matter volume in several subcortical structures moderated extinctionanxiety associations. Using a modeling approach, we identify computational mechanisms linking threat learning and anxiety severity and their neuroanatomical substrates.
\end{abstract}




\section{Introduction}

Threat learning encompasses processes that rapidly generate associations between neutral stimuli and aversive outcomes. Influential neurodevelopmental theories implicate age-related variations in the mechanisms underlying such conserved associative-learning processes in anxiety symptoms ${ }^{1,2}$. Computational learning theory offers tools to quantify associative learning dynamics as they relate to variations in these mechanisms. Here, we study physiological data recorded during a threat learning paradigm in a large sample $(\mathrm{N}=251)$ featuring a wide range normative to pathological anxiety severity across childhood to adulthood. We use these data to uncover links among threat learning processes, their neuroanatomical substrates, and anxiety severity via latent learning parameters estimated by reinforcement learning models.

Neural circuits have evolved to allow rapid learning of threat associations following encounters with danger ${ }^{3,4}$. Through threat conditioning, a neutral stimulus rapidly comes to elicit fear responding in anticipation of danger ${ }^{3}$. Through extinction, such conditioned anticipatory responding is attenuated if the stimulus no longer predicts the occurrence of threat ${ }^{5}$. Extensive research across species implicates conserved neural circuitry in these processes, highlighting their adaptive value ${ }^{6-13}$.

At the same time, aspects of these processes could prove maladaptive. Thus, influential theories link variations in threat learning to the emergence and persistence of excessive threatanticipatory fear responses which are central in expression of anxiety symptoms $s^{1,2,14-16}$. Moreover, maturational processes in the circuitry supporting such learning may underlie the emergence of anxiety symptoms in childhood and adolescence ${ }^{17-23}$. However, studies in youth and adults attempting to link variations in threat learning and anxiety symptoms yield 
inconsistent findings ${ }^{2,14,24}$, hindering neuroscience research on normal and abnormal threat learning mechanisms.

Failure to detect robust and replicable associations between threat learning and anxiety symptoms could reflect limitations of standard analytic methods that are not designed to capture associative learning dynamics ${ }^{8,25}$. Such limitations could potentially be overcome through applications of computational learning theory, which provides a mathematical framework for quantifying the temporal dynamics of associative learning processes, as indexed with latent variables $^{11,13,26,27}$. Based on the measurement of prediction errors between expected and experienced outcomes, associative learning models were initially applied to reward learning ${ }^{26,28,29}$. Recent studies extend this approach to model associations between threat cues and aversive outcomes. These include studies in healthy subjects and in patients with posttraumatic stress disorder, identifying cumulative cue-associability over time as an important learning parameter that also relates to symptoms ${ }^{12,30-33}$. This approach has yet to be applied to study the mechanisms that link individual differences in threat learning processes and anxiety symptoms $s^{1,2,14}$.

Here, we address this gap by applying reinforcement learning models to skin conductance response (SCR) data recorded during a threat learning paradigm, in conjunction with structural brain imaging, to quantify age-dependent associations among threat learning processes, neuroanatomy, and anxiety severity. We complement and extend prior work in several ways. First, we study a sample that includes individuals with and without anxiety disorders falling along a wide age span, from age 8-50 years. This sample contains wide ranges of anxiety symptoms and age, which increase statistical power to detect associations among age, anxiety, and threat learning indices. Second, previous work modeled cue responding over many 
reinforcement trials (e.g., $>60$ trials) ${ }^{12,30,31}$, which may more closely model the expression of conditioned responses. Here, we focus instead on the rapid learning of threat associations that take place during a shorter schedule ${ }^{3}$. Third, we model four learning effects: threat conditioning, threat generalization, safety learning, and threat extinction. This provides tests of different theories that link mechanistic variations in these specific processes to the severity of anxiety ${ }^{1,8,23,34-36}$. Finally, we use data from a relatively large sample $(\mathrm{N}=215)$ of medication-free subjects, enabling parameter estimation with greater accuracy and over a large model space. We hypothesize that anxiety severity is associated with latent learning parameters ${ }^{1,2}$; that age moderates specifically the association between extinction learning and anxiety ${ }^{19}$; and that morphometry features in subcortical structures and prefrontal cortex moderate associations between learning parameters and anxiety ${ }^{37,38}$.

\section{Methods}

\section{Participants}

Date from a sample of 351 individuals were initially considered. Due to our focus on model fitting with trial-by-trial data and the noisy nature of SCR data, we excluded individuals with excessive missing data, with similar exclusion proportion to prior work ${ }^{12}(\mathrm{n}=136$; see Supplement). This led to a final sample of $\mathrm{N}=215$, which included 104 healthy participants (53 females; ages 8-44 years) and 111 medication-free participants with anxiety disorders (63 females; ages 8-50 years). Healthy and anxiety groups did not differ in age, sex, or IQ, $p \mathrm{~s}>05$. All participants were recruited from the community as paid volunteers for research at the National Institute of Mental Health (NIMH). Written informed consent was obtained from adult ( $\geq 18$ years) participants as well as parents, and written assent was obtained from youth. 
Procedures were approved by the NIMH Institutional Review Board. Analyses on raw psychophysiology data and neuroanatomy data from this sample have been previously reported $^{34,39-41}$. The current study reports novel analyses of these data.

\section{Anxiety severity}

All participants were interviewed by trained clinicians using semi-structured clinical interviews; see Supplement. Anxious patients were required to meet criteria for generalized, social, and/or separation anxiety disorder. Healthy participants did not meet criteria for any psychiatric diagnosis. See supplement for exclusionary criteria. To assess current anxiety symptom severity, youth and adult completed standard anxiety questionnaires. Youth completed the Screen for Child Anxiety Related Emotional Disorders (SCARED) ${ }^{42}$, and adults completed the trait subscale of the STAI ${ }^{43}$, within 3 months of the task. The SCARED is a child- and parent-report measure comprising 41 items assessing recent anxiety symptoms and possessing strong psychometric properties ${ }^{42,44}$; to reduce informant differences, child- and parent-report scores were averaged ${ }^{45,46}$. The $\mathrm{STAI}^{43}$ consists of 20 items relating to general anxious moods and possesses strong psychometric properties ${ }^{47}$. To combine these anxiety measures, we Ztransformed each measure within its respective age sample; these Z-scores were then combined across samples and used in analyses ${ }^{48}$. These anxiety severity scores manifested a unimodal, continuous distribution across the sample (see Fig. S1).

\section{Threat conditioning and extinction task}

The task involves rapid, uninstructed Pavlovian conditioning and extinction of threat associations $^{3}$. The task has previously been found effective (i.e., produce conditioning while maintaining a low dropout rate) among individuals with anxiety and healthy participants from 
both youth and adult populations ${ }^{21,35,39-41,49-51}$. In the task (see Fig. 1), photographs of two women displaying neutral expressions ${ }^{52}$ served as the conditioned threat and safety stimuli (CS+ and CS-, respectively). The unconditioned stimulus (UCS) was a 1s presentation of the same actress displaying fear and co-occurring with a $95 \mathrm{~dB}$ female scream delivered via headphones, co-terminating with the CS+. During pre-conditioning, each CS was presented 4 times. During conditioning, each CS was presented 10 times, and the CS+ was followed by the UCS with an $80 \%$ reinforcement schedule. During extinction, the CSs were each presented eight times and never reinforced. In all phases, the duration of the CS+ and CS- were presented for 8 seconds, but shortened to 7s when UCS occurred. See Supplement for additional details. Skin conductance, electromyography, and electrocardiography were recorded. Due to our focus on skin conductance responses as indexing conditioned anticipatory fear responses ${ }^{8}$, the other measures are not analyzed in the current report.

Skin conductance. Skin conductance was recorded at 1,000Hz using PsyLab from two $\mathrm{Ag} / \mathrm{AgCl}$ electrodes from the medial phalanx of the middle and ring non-dominant-hand fingers. In line with prior research, skin conductance response (SCR) was determined by the square-roottransformed difference between base-to-peak amplitude within 1-5s after stimulus onset ${ }^{31-}$ 33,39,53,54. To generate more accurate estimates from trial-by-trial data, we next cleaned trial-level data as reported in the Supplement.

\section{Raw SCR data}

Raw SCR data by trial and task phase are depicted in Figure 1. During the conditioning phase, rapid CS+ threat conditioning (acquisition of conditioned fear response) was noted; response to the CS- was characterized by generalization of conditioned fear response (increased response despite no reinforcement) and safety learning (diminishing response through continued 
non-reinforcement). During the extinction phase, rapid extinction (diminishing response) was noted for both stimuli. Statistics on these data are reported in the Results section. As noted, perturbations in these processes have been suggested to contribute to the emergence and persistence of anxiety symptoms ${ }^{1,2,14,55-57}$. Specifically, facilitated threat conditioning ${ }^{58}$, greater threat generalization ${ }^{55,57,59}$, and slower safety learning ${ }^{22,23}$ have each been theorized to lead to underlie anxiety symptoms. Further, threat extinction processes have been implicated in both anxiety symptoms and exposure-based treatment for anxiety ${ }^{5,19,22,57}$. Due to the potential relevance of these learning processes to anxiety, and as they were evident in the data, we used reinforcement modeling to examine them.

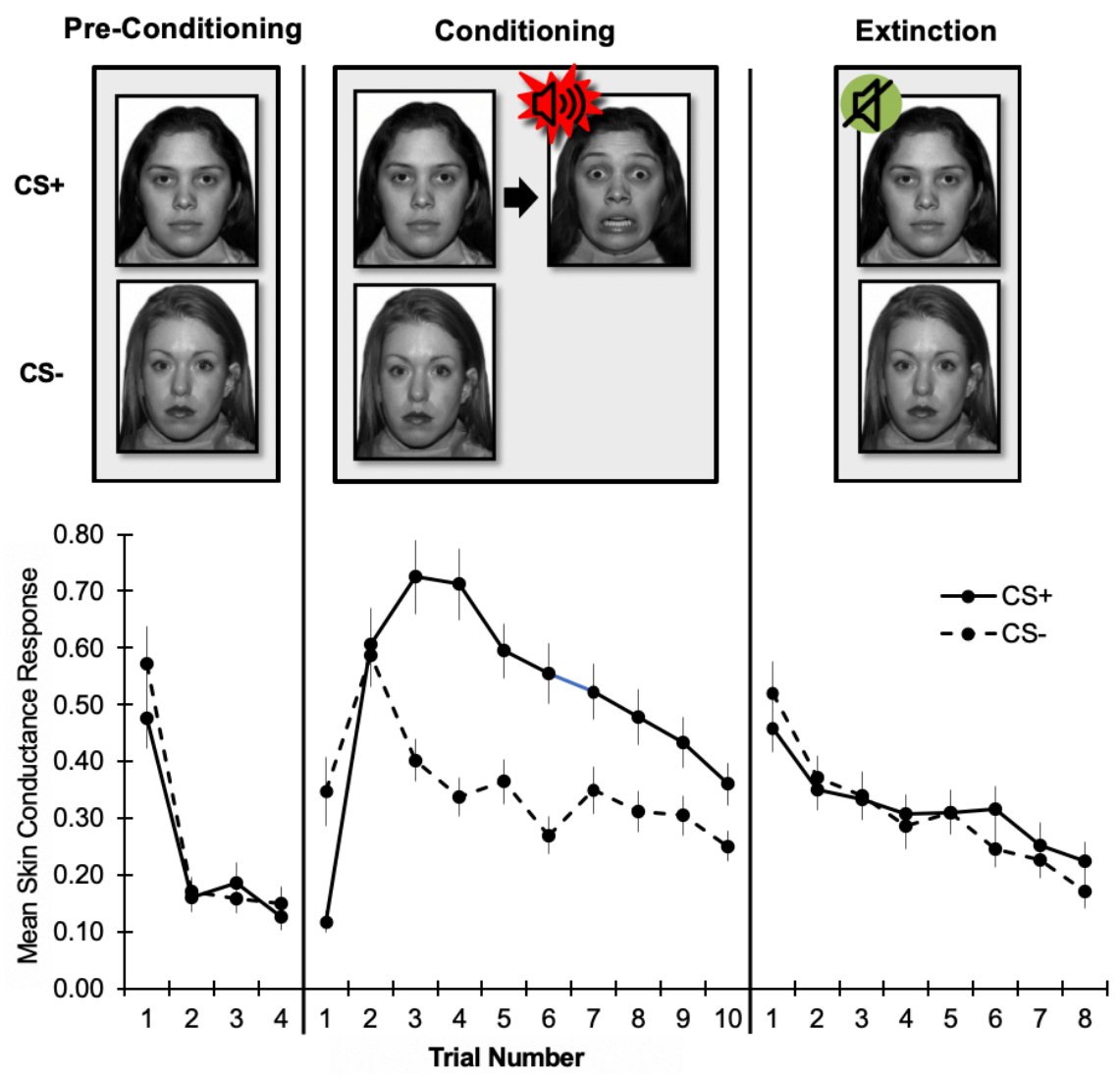


Figure 1. Top: Schematic representation of the threat learning paradigm. During the preconditioning phase, the designated threat (CS+) and safety (CS-) stimuli were presented without reinforcement. During the conditioning phase, the CS+ was paired with a fearful face co-terminating with a scream (UCS); the CS- was never reinforced. During the extinction phase, both CS+ and CS- were not reinforced by the UCS.

Bottom: Mean raw skin conductance response for the CS+ and CS- by task phase and trial.

Note: Trial number indicates the nth trial for that stimulus. However, the CS+ and CStrials were presented in counterbalanced order throughout the task. CS = conditioned stimulus; UCS = unconditioned stimulus. Error bars indicate one standard error of the mean.

\section{Reinforcement learning models}

In previous work on this data we used standard statistical models to assess the effects of anxiety on conditioning ${ }^{34}$. In this manuscript, the goal was to explore dynamic learning processes that could account for the model-agnostic effects seen in the previous study. To model these dynamic processes during threat conditioning and extinction, we fit separately for each participant multiple reinforcement learning models to the trial-level SCR data. The models learn, trial by trial, the value of the CS based on the presence or absence of the aversive stimulus (UCS). See Fig. S2 for histograms depicting model estimates in the sample.

1. Conditioning phase: $\mathrm{CS}+$ threat conditioning. First, we modeled threat conditioning whereby the CS+ comes to elicit a threat-anticipatory response via reinforcement by the UCS. 
We fit nine reinforcement learning models to $\mathrm{CS}+$ conditioning data that varied in the number of parameters used to model the psychophysiological responses, as detailed next. Parameters were estimated and optimized for each participant separately using the fminsearch function in MATLAB by minimizing the sum of squares error between the predicted and measured SCR to the $\mathrm{CS}^{35}$. We then compared the model fits to the actual data per participant and across the sample to choose the best-fitting model.

All models were initially based on a Rescorla-Wagner (RW) model ${ }^{27}$, whereby at trial $t+1$, the value $v_{C S+}$ of the CS+ is updated based on its value at the preceding trial $v_{C S+}(t)$ plus the prediction error obtained at trial $t$ (corresponding to the difference between the value of the UCS at that trial and the expected value, multiplied by the learning rate parameter $\alpha$ ):

$$
v_{C S+}(t+1)=v_{C S+}(t)+\alpha\left[r(t)-v_{C S+}(t)\right]
$$

where $r(t)$ is the SCR to the UCS on trial $t$. For each trial, the updated predicted SCR to the $\mathrm{CS}+$ is defined as $v_{C S+}(t+1)$.

The threat conditioning data displayed additional dynamics features that would not be captured by the base RW model (Fig. 1). Therefore, we explored additional learning mechanisms, which led to 8 models (see Table 1). First, we fit a learning inertia term ${ }^{60}$, which assumed that the effect of the prediction error accumulated across $m$ recent trials. Specifically, if we define the prediction error on trial $t$ as $\delta(t)=r(t)-v_{C S+}(t)$, the learning inertia term updated the value using $v_{C S+}(t+1)=v_{C S+}(t)+\alpha \delta^{i n}$, where

$$
\delta^{i n}(t)=\sum_{k=0}^{k_{m}} \delta(t-k)
$$

The value $k_{m}=2$ maximized the fit across all participants. Qualitatively this model improved the fit, but we found only indeterminate statistical evidence to support it. 
Second, we examined Bayesian learning rate decay. This assumes that the learning rate starts high and then diminishes over trials according to the information that has been accumulated $^{61}$. In this model variant, $\alpha$ was a free parameter and the learning rate on each trial was given by:

$$
\alpha(t)=\frac{\alpha}{\operatorname{sqr} t(t)}
$$

This variant has no additional free parameters over the base RW model.

Third, we examined habituation of the conditioned response (diminished response over trials despite reinforcement). Accordingly, we fit a multiplicative exponential decay term, which resulted in an SCR estimate given by:

$$
v_{C S+}^{h a b}(t)=v_{C S+}(t) e^{-\varphi\left[t-t_{0}\right]^{+}}
$$

When we fit this model, we estimated $v_{C S+}(t)$ using equation 1 , and then multiplied the estimated values by the habituation term given in equation 4 . This model had an additional parameter for each participant, $\varphi$, which controlled the rate of habituation. The rectified linear operator, $[\mathrm{x}]^{+}$, returns 0 for negative values and $\mathrm{x}$ for positive values. We also estimated a single value of $t_{0}$ across all participants, as it was clear that the habituation process did not start in the first conditioning trial. We found that $t_{0}=2$ was optimal and significantly improved the pooled model fit across participants.

Lastly, in addition to the 8 aforementioned models, we fit a RW-Pearce-Hall (PH) "hybrid" model to the data, given findings on its relevance to threat learning processes ${ }^{12,30-32}$. This model utilizes equation 1 for the value update with absolute value of the prediction error. However, in the PH model the learning rate is adaptive, and updated on each trial as:

$$
\alpha=\alpha(1-\gamma)+\gamma|\delta(t)|
$$


The variable $\gamma$ is a free parameter that controls the rate of update of the learning rate. The hybrid model, therefore, has 2 parameters $(\alpha, \gamma)$.

As these model structures were independent, we fit 9 models (see Table 1), where model 1 was the base RW model; model 2 included a prediction-error inertia term; model 3 assumed a Bayesian learning-rate decay; model 4 included a habituation term; model 5 included the inertia term and Bayesian learning-rate decay; model 6 included inertia and habituation terms; model 7 included Bayesian learning-rate decay and habituation; model 8 included inertia, Bayesian learning-rate decay, and habituation; and model 9 was the RW-PH hybrid model. See Table 1. We calculated the Bayesian Information Criterion $(\mathrm{BIC})^{62}$, which offers a trade-off between model fit and model complexity, for each model, and then carried out $t$-tests on the BIC across the sample to identify the best-fitting model. Parameters from the best fitting model were then used in analyses of brain structure, anxiety symptoms, and age.

\begin{tabular}{|c|c|c|}
\hline Model & Model specification & Free parameters \\
\hline 1. RW & $v_{C S+}(t+1)=v_{C S+}(t)+\alpha \delta$ & $\alpha$ \\
\hline 2. RW with inertia & $\begin{array}{l}v_{C S+}(t+1)=v_{C S+}(t)+\alpha \delta^{i n}, \text { whereby } \delta^{i n}(t)= \\
\sum_{k=0}^{k_{m}} \delta(t-k), \text { with } m=\text { number of recent trials }\end{array}$ & $\alpha$ \\
\hline $\begin{array}{l}\text { 3. RW with Bayesian } \\
\text { learning-rate decay }\end{array}$ & $v_{C S+}(t+1)=v_{C S+}(t)+\alpha \delta$, whereby $\alpha(t)=\frac{\alpha}{\operatorname{sqrt}(t)}$ & $\alpha$ \\
\hline $\begin{array}{l}\text { 4. RW with } \\
\text { habituation }\end{array}$ & $\begin{array}{l}v_{C S+}(t+1)=v_{C S+}(t)+\alpha \delta, \text { with } v_{C S+}(t) \text { multiplied } \\
\text { by } e^{-\varphi\left[t-t_{0}\right]^{+}} \text {after update }\end{array}$ & $\alpha, \varphi$ \\
\hline $\begin{array}{l}\text { 5. RW with inertia and } \\
\text { Bayesian learning- } \\
\text { rate decay }\end{array}$ & $\begin{array}{l}v_{C S+}(t+1)=v_{C S+}(t)+\alpha \delta^{i n}, \text { whereby } \alpha(t)=\frac{\alpha}{\operatorname{sqrt}(t)}, \\
\text { and } \delta^{i n}(t)=\sum_{k=0}^{k_{m}} \delta(t-k), \text { with } m=\text { number of recent } \\
\text { trials }\end{array}$ & $\alpha$ \\
\hline
\end{tabular}




\begin{tabular}{|c|c|c|}
\hline $\begin{array}{l}\text { 6. RW with inertia and } \\
\text { habituation }\end{array}$ & $\begin{array}{l}v_{C S+}(t+1)=v_{C S+}(t)+\alpha \delta^{i n}, \text { whereby } \delta^{i n}(t)= \\
\sum_{k=0}^{k_{m}} \delta(t-k), \text { with } m=\text { number of recent trials, and } \\
v_{C S+}(t) \text { multiplied by } e^{-\varphi\left[t-t_{0}\right]^{+}} \text {after update }\end{array}$ & $\alpha, \varphi$ \\
\hline $\begin{array}{l}\text { 7. RW with Bayesian } \\
\text { learning-rate decay } \\
\text { and habituation }\end{array}$ & $\begin{array}{l}v_{C S+}(t+1)=v_{C S+}(t)+\alpha \delta, \text { whereby } \alpha(t)=\frac{\alpha}{\operatorname{sqr}(t))^{\prime}} \\
\text { and } v_{C S+}(t) \text { multiplied by } e^{-\varphi\left[t-t_{0}\right]^{+}} \text {after update }\end{array}$ & $\alpha, \varphi$ \\
\hline $\begin{array}{l}\text { 8. RW with inertia and } \\
\text { Bayesian learning- } \\
\text { rate decay, and } \\
\text { habituation }\end{array}$ & $\begin{array}{l}v_{C S+}(t+1)=v_{C S+}(t)+\alpha \delta^{i n}, \text { whereby } \alpha(t)=\frac{\alpha}{\operatorname{sqrt}(t)} \\
\text { and } \delta^{i n}(t)=\sum_{k=0}^{k_{m}} \delta(t-k), \text { with } m=\text { number of recent } \\
\text { trials, and } v_{C S+}(t) \text { multiplied by } e^{-\varphi\left[t-t_{0}\right]^{+}} \text {after update }\end{array}$ & $\alpha, \varphi$ \\
\hline 9. RW-PH hybrid & $\begin{array}{l}v_{C S+}(t+1)=v_{C S+}(t)+\alpha \delta, \text { whereby } \alpha=\alpha(1-\gamma)+ \\
\gamma|\delta(t)|\end{array}$ & $\alpha, \gamma$ \\
\hline
\end{tabular}

Table 1. Specifications and estimated free parameters for each model fit to the threat conditioning data from the conditioning phase. In all models, prediction error is defined as: $\delta(t)=r(t)-v_{C S+}(t)$.

Note: $\mathrm{RW}=$ Rescorla-Wagner base model; $t=$ current trial; $v_{C S+}(t+1)=$ predicted skin conductance response to $\mathrm{CS}+$ at trial $t+1 ; r(t)=$ measured skin conductance response to UCS at trial $t$.

2. Conditioning phase: CS- threat generalization and safety learning. As can be seen in the conditioning phase data (Fig. 1), participants rapidly generalized a fear response to the CS(i.e., showed an increase in response to CS- despite non-reinforcement), and learned its safety value as it was continually non-reinforced (i.e., safety learning). A standard RW model will not capture conditioning to the CS-, as this cue was never reinforced. Generalization of the conditioned response to the CS- and safety learning could be captured by a model that tracks the psychophysiological responses to the CS+ and "projects" them onto the CS-. This model 
contains four parameters: a learning rate (equation 1) and a habituation term (equation 4) for each of the CS- and CS+. This uses the best-fitting model for the CS+ (see Results) with an additional value equation for the CS-. In this model, when a UCS was delivered, the value for the $\mathrm{CS}+$ was updated according to equation 1 and, in addition, a value for the CS- was updated using

$$
v_{C S-}(t+1)=v_{C S-}(t)+\alpha_{C S-} \delta(t)
$$

Note that $\delta(t)$ was only calculated in CS + trials when a UCS was delivered. There was no update in CS- trials. Fourteen of the 215 participants had fewer than 3 CS- trials with nonzero data and were thus excluded from analyses.

3. Extinction phase: CS+ threat extinction learning. Next, we modeled $\mathrm{CS}+$ threat extinction rates, whereby participants learn to omit anticipatory responses to the CS+ as this cue is no longer reinforced by the UCS. Data indicated that extinction learning follows exponential decay. Accordingly, this model considered one parameter for the decay rate, using equation 1 to model $\mathrm{CS}+$ data during extinction.

Of note, the learning processes studied here manifest over few trials, which could make parameter estimates noisy. To accommodate for that, we use data from a large number of participants. Because our hypotheses were tested using hierarchical models, variance in parameter estimates at the first level can be compensated for with additional participants, when testing hypotheses at the second level.

\section{Brain imaging}

Analyses tested associations among threat learning parameters and imaging measures. MRI images $\left(1 \mathrm{~mm}^{3}\right)$, acquired on a 3-Tesla MR750 GE scanner with a 32-channel head coil (see Supplement for acquisition parameters), were collected from 148 of the 215 participants 
$(69 \% ; 81$ females, Mage $=18.38$ years $)$. MRI data for a larger sample containing these participants appear in previous reports using different analyses ${ }^{34,63,64}$. FreeSurfer (version 6.0, http://surfer.nmr.mgh.harvard.edu) was used for processing (see Supplement). Statistical tests were performed using PALM (Permutation Analysis of Linear Models) ${ }^{65}$. Surface-based analyses considered whole-brain cortical thickness using the TFCE (threshold-free cluster enhancement) statistic ${ }^{66}$, with a vertex-wise familywise-error (FWE)-corrected threshold of $p<0.05$. Analyses of subcortical volumes generated by FreeSurfer considered gray matter volume (GMV) with an FWE-corrected threshold of $p<0.05$. For each morphometry measure, analyses included global whole-brain estimates of the measure (global average thickness, total intracranial volume) as nuisance, as recommended in prior research ${ }^{67}$. Sex was also used as a nuisance variable given reported sex differences in brain structure ${ }^{17,34,68}$. See Supplement for additional details. To facilitate reproducibility, the full analysis pipeline has been previously published ${ }^{34}$.

\section{Data analysis}

Analyses tested associations among threat learning parameters, morphometry measures, anxiety severity, and age. Specifically, for each of the three processes modeled (see above), we first identified the model best accounting for the process (e.g., $\alpha$ in threat conditioning). For each estimated parameter in this model, we next conducted two analyses. First, we examined whether the estimated learning parameter was associated with anxiety severity, as moderated by age, by testing the Parameter $\times$ Age interaction on anxiety scores. Second, we examined whether brain structure was associated with the learning parameter, by testing the StructurexAnxiety $\times$ Age main and interaction effects on parameter values. 
All analyses used the general linear model and set significance at 0.05 . In addition to the aforementioned corrections for multiple testing, within each of the three processes studied we further applied a Bonferroni correction to statistical tests to account for the number of parameters tested in the model; all reported $p$-values reflect these corrections.

\section{Results}

\section{Raw SCR data}

Raw SCR data by trial and phase are depicted in Figure 1. Repeated-measures ANOVA of SCR data with Phase (conditioning, extinction) and CS (CS-, CS+) as within-subject factors and anxiety severity (Z-scores) and age (years) as between-subject factors, indicated a significant Phase $\times$ CS interaction, $F(1,212)=10.97, p=0.001$. Follow-up analyses indicated greater response to $\mathrm{CS}+$ than $\mathrm{CS}$ - during conditioning, $F(1,212)=24.24, p<0.001$, but not during extinction, $F(1,212)=0.24, p=0.63$, demonstrating successful conditioning and extinction in the task. Anxiety and age did not significantly moderate the Phase $\times \mathrm{CS}$ interaction. Additional lower-order effects are reported in supplement.

\section{Reinforcement models}

\section{Conditioning phase: $\mathrm{CS}+$ threat conditioning}

Model fit. Fig. 2A depicts empirical and modeled data for each of the 9 models fit to the CS+ conditioning data. Fig. 2B depicts for descriptive purposes, for each model, the mean correlation between empirical and modeled data across the sample. To determine which model provided the best fit, we ranked their BIC values using $t$-scores from dependent-samples $t$-tests. Models 7 (RW with Bayesian learning-rate decay and habituation term) and model 8 (RW with inertia 
term, Bayesian learning-rate decay, and habituation term) provided better fit than the other models (all $t \mathrm{~s} \geq 3.89$, and $t \mathrm{~s} \geq 5.48$, respectively). While model 8 provided a slightly better fit than $7, t=1.35$, the latter was simpler, and thus we used parameters from model 7 (threat learning rate, $\alpha$; and habituation rate, $\varphi$ ) in subsequent analyses. 

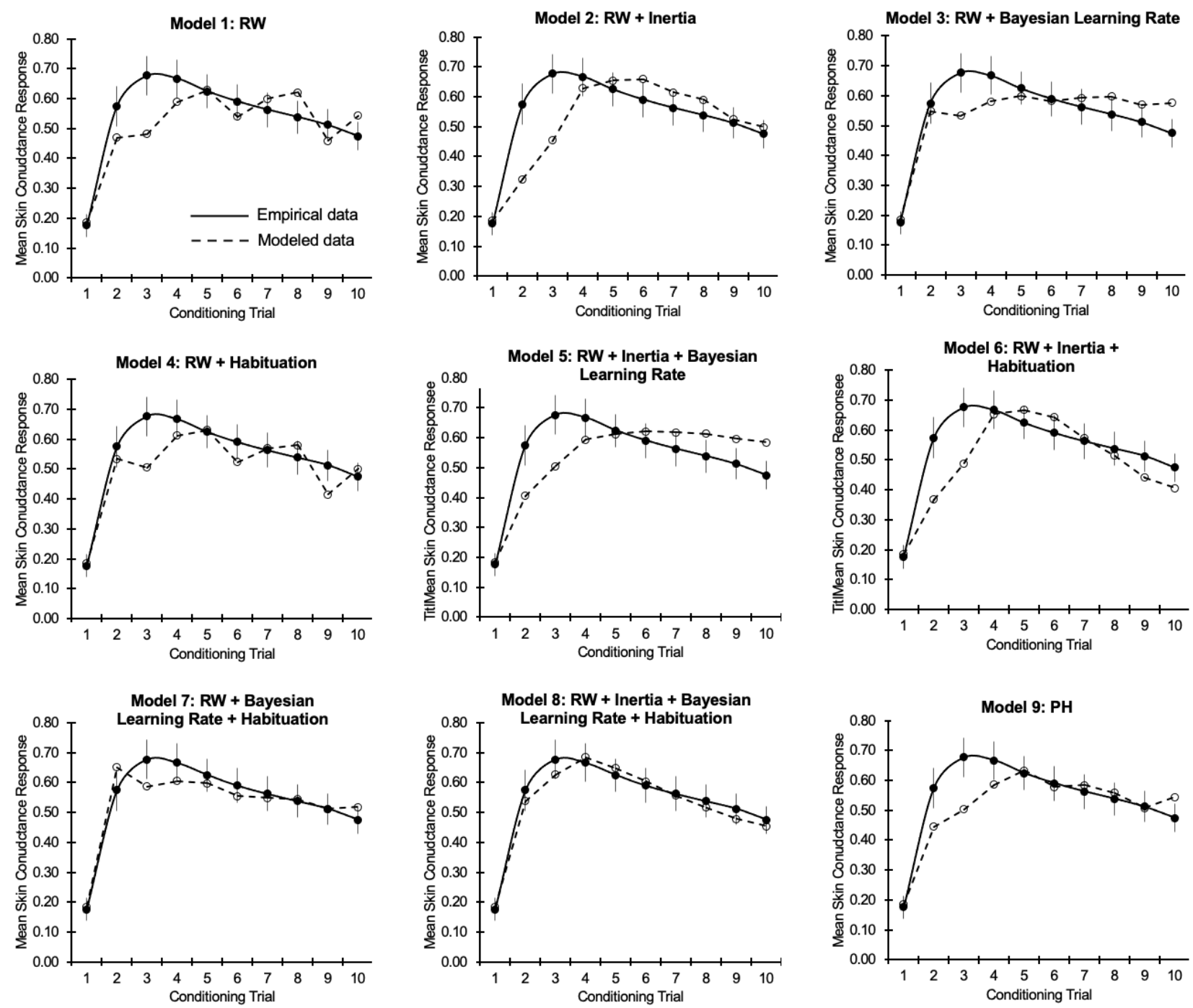

\section{B. Conditioning: Mean Correlations between Empirical and Modeled Data}

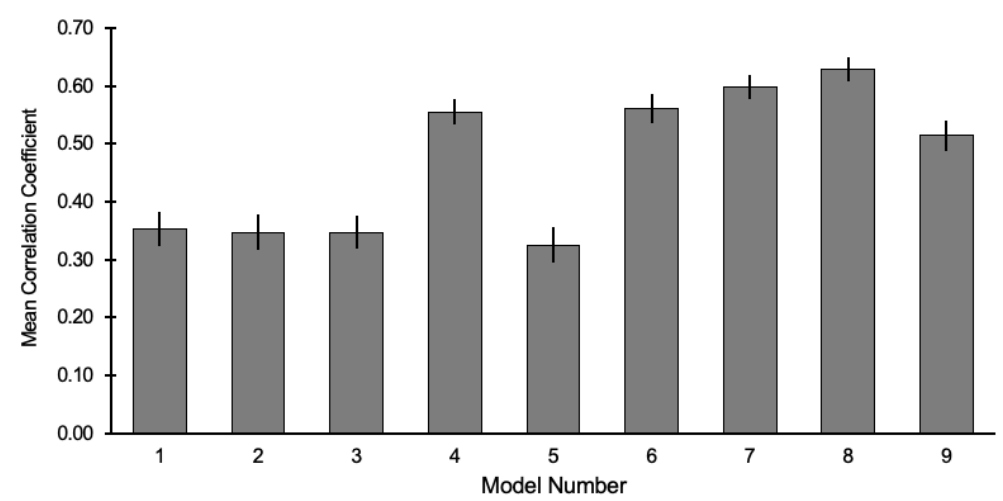


Fig. 2. CS+ threat conditioning model fit. A) Graphs depict empirical skin conductance data (full line; bars indicate one standard error of the mean) and fitted data (dashed line) for each of the models fitted to CS + conditioning data. B) For each model, the bar depicts the mean correlation coefficient between the empirical and modeled data (error bars reflect one standard error of the mean, all mean coefficients are significantly different than $0, p s<0.001)$. Model numbers correspond to specification in (A) and to Table 1 .

Anxiety and age. Threat learning rate and habituation rate during conditioning did not differ by anxiety severity or age, $\beta \mathrm{s}<0.06, p \mathrm{~s}>0.41$.

Brain structure. No effects of brain structure on threat learning rate were noted. Habituation rate correlated positively with right thalamus GMV, $\beta=0.28, p=0.008$ (corrected); see Fig. 3. No other effects were observed, all $p \mathrm{~s}>0.05$.

\section{Conditioning: Thalamus Gray Matter Volume and CS+ Habituation Rate}
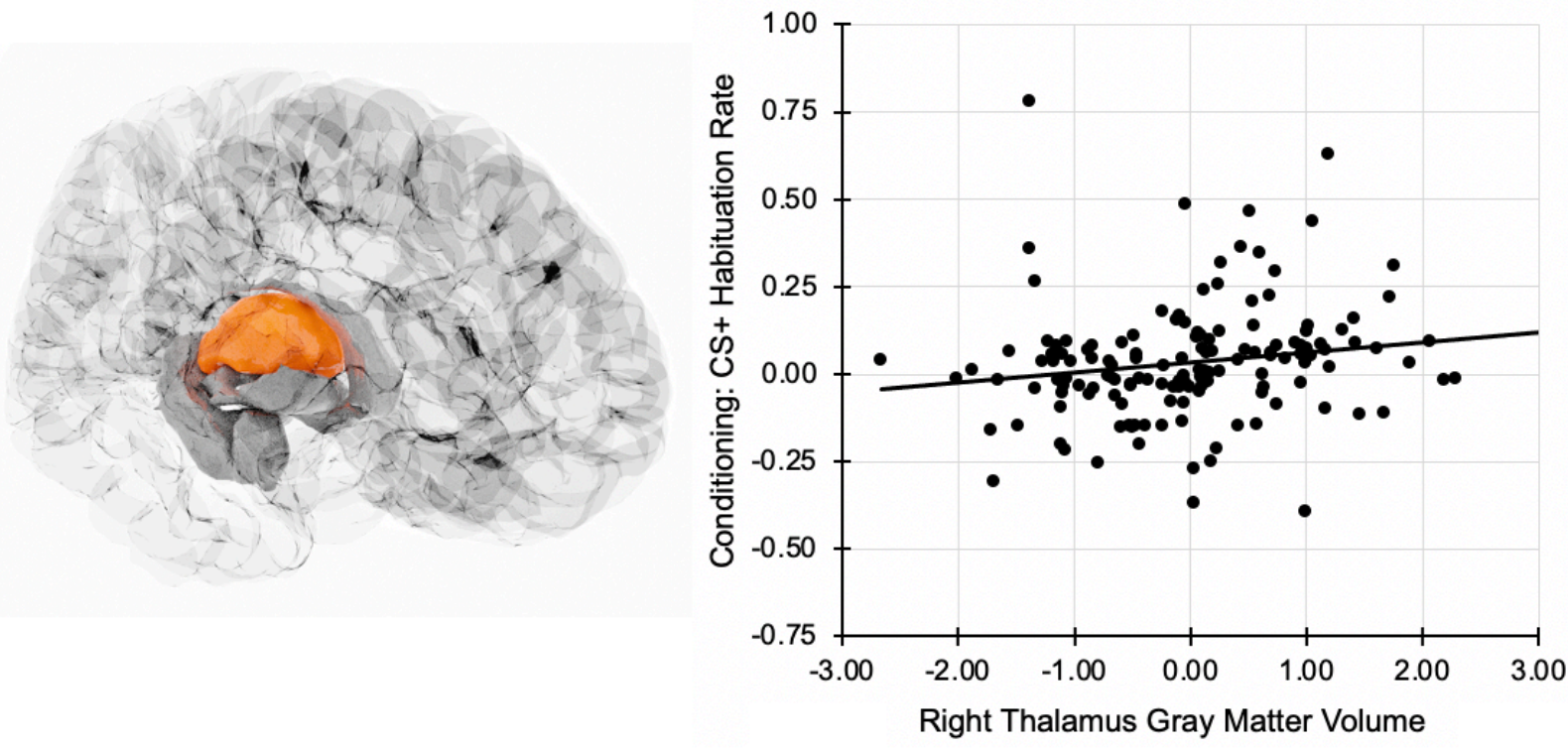
Figure 3. Conditioning: CS+ habituation rate. Scatterplot depicts correlation between right thalamus gray matter volume and $\mathrm{CS}+$ habituation rate during conditioning. Data shown are residuals after controlling for nuisance and other effects of interest.

\section{Conditioning phase: CS- threat generalization and safety learning}

Model fit. For the CS- data, we fit the best-fitting model as for the CS+. This model captured the generalization of fear response to the non-reinforced CS- and decay in response to CS- that reflects learning its safety value; see Fig. 3A. The average correlation across the sample between the modeled and empirical data was $r=0.54, p<0.001$.

Anxiety and age. Anxiety severity was associated with both CS- threat generalization rate, which captures initial transfer of CS+ threat learning to the CS- cue, and CS- safety learning rate, which captures the reduction in responding through non-reinforcement. CSthreat generalization rate interacted with age in predicting anxiety severity, $\beta=0.42, p=0.028$ (corrected). To interpret this developmental effect, we tested the generalization-anxiety simple correlation separately within each of three age-based groups broadly corresponding to pre-adolescence (age $<13$ years, $n=74)$, adolescence $(13 \leq$ age $<18$ years, $n=57)$, and adulthood (age $>18$ years, $n=69$ ); see Fig 3B. Threat generalization rate and anxiety showed a significant negative correlation in the pre-adolescence group, $r(73)=-0.29, p=0.011$; this correlation was not significant in the adolescence or adult age group, $r(56)=-0.21, p=0.11$, and $r(68)=0.18$, $p=0.15$, respectively. The correlation in the adult group was significantly different from the correlation in the adolescent group, $Z=2.15, p=0.032$, and in the pre-adolescent group, $Z=2.81, p=0.005$. Thus, generalization rate was associated with anxiety only in younger age. 
In addition, CS- safety learning rate was correlated with anxiety severity, such that slower safety learning was associated with greater anxiety, regardless of age, $\beta=-0.22$, $p=0.004$ (corrected); see Fig. 3C. No other effects were observed.
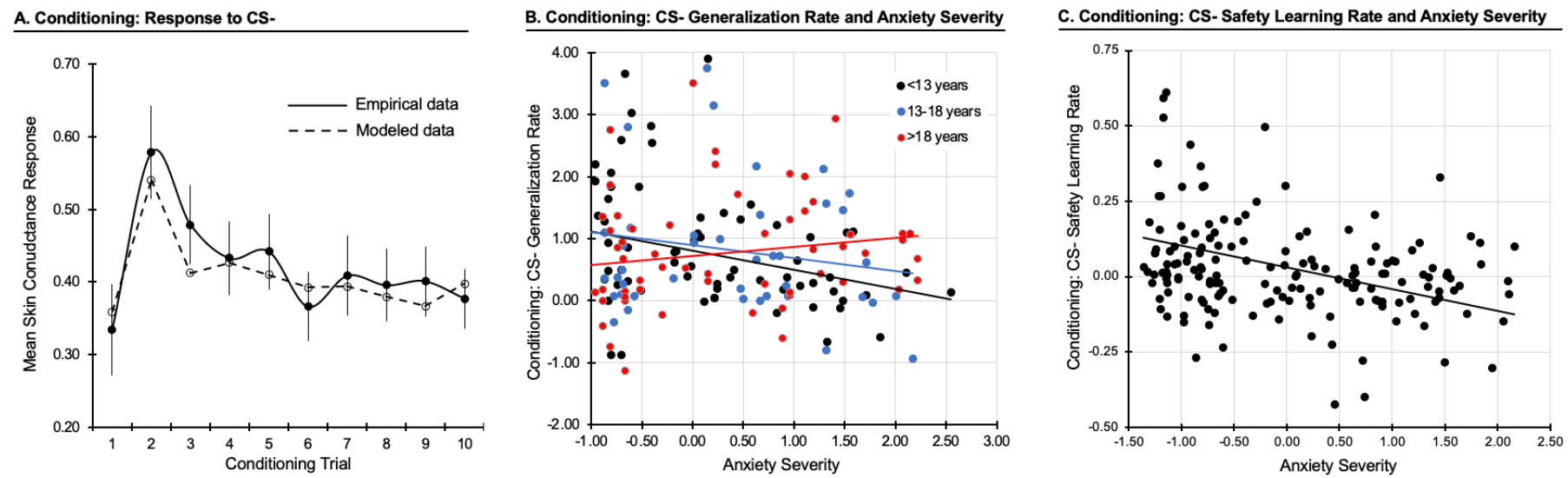

Figure 3. A) Graph depicts empirical skin conductance data (full line; bars indicate one standard error of the mean) and fitted data (dashed line) for the model fit to the CSconditioning data, which includes threat generalization rate and safety learning rate terms. B) Scatterplot depicts age moderation of association between CS- threat generalization rate during conditioning and anxiety severity, decomposed into three age groups. C) Scatterplot depicts correlation between anxiety severity and CS- safety learning rate during conditioning. Data shown are residuals after controlling for nuisance and other effects of interest.

Brain structure. No significant effects were observed, $p \mathrm{~s}>0.05$. 


\section{Extinction phase: $\mathrm{CS}+$ threat extinction}

Model fit. $\mathrm{CS}+$ threat extinction featured exponential decay. Empirical data and modeled data are depicted in Fig. 4A. The average correlation across the sample between the modeled and empirical data was $r=0.52, p<0.001$.

Anxiety and age. Threat extinction rate correlated with anxiety severity, $\beta=-0.19$, $p=0.013$ (corrected), such that slower extinction was associated with greater anxiety, see Fig. 4B. No other effects were observed, $p \mathrm{~s}>0.05$.

Brain structure. GMV in several subcortical structures moderated the association between $\mathrm{CS}+$ extinction learning rate and anxiety severity, including brain stem, bilateral ventral diencephalon (which includes hypothalamus and midbrain), bilateral caudate nucleus, and left putamen, all $\beta \mathrm{s} \geq 1.06, p \mathrm{~s} \leq 0.013$ (corrected). Decomposition of this interaction revealed a consistent pattern, whereby as GMV was smaller, slower threat extinction was associated with greater anxiety severity; see Fig. 4C for a representative graphical depiction of this effect (brain stem GMV). 


\section{A. CS+ Extinction Model Fit}

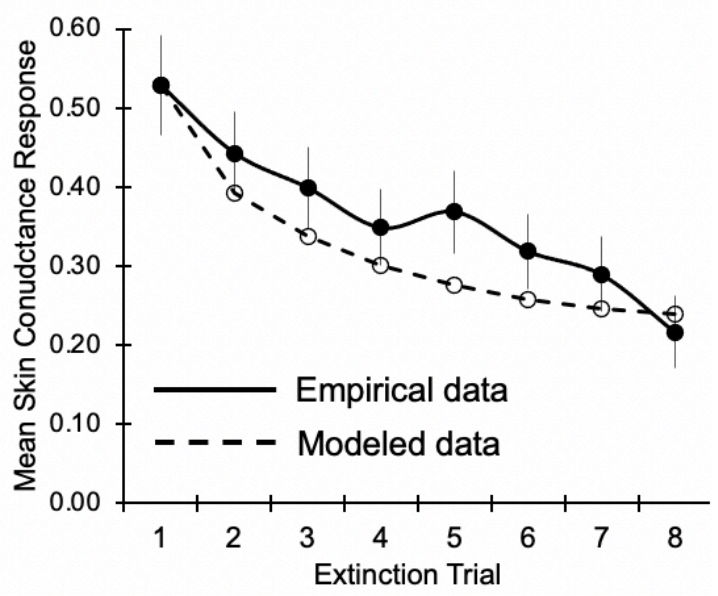

B. CS+ Extinction Rate and Anxiety Severity

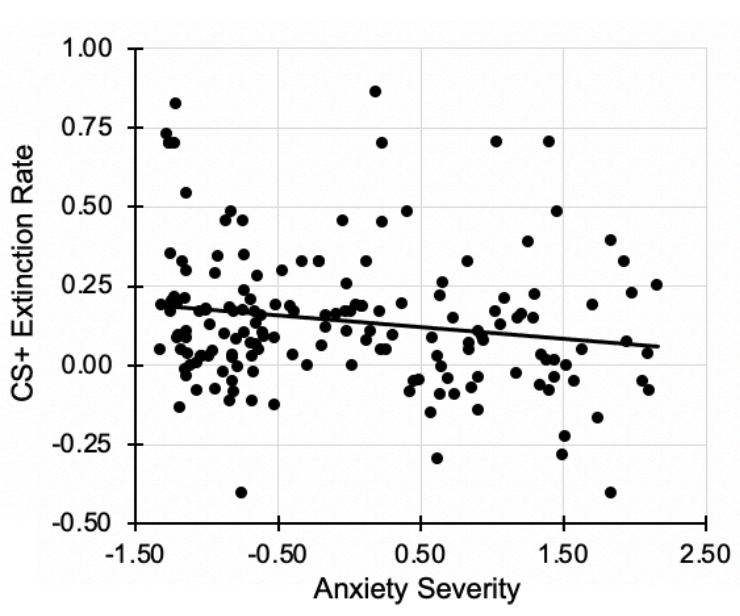

\section{Subcortical Volume Moderates Link between CS+ Extinction Rate and Anxiety Severity}
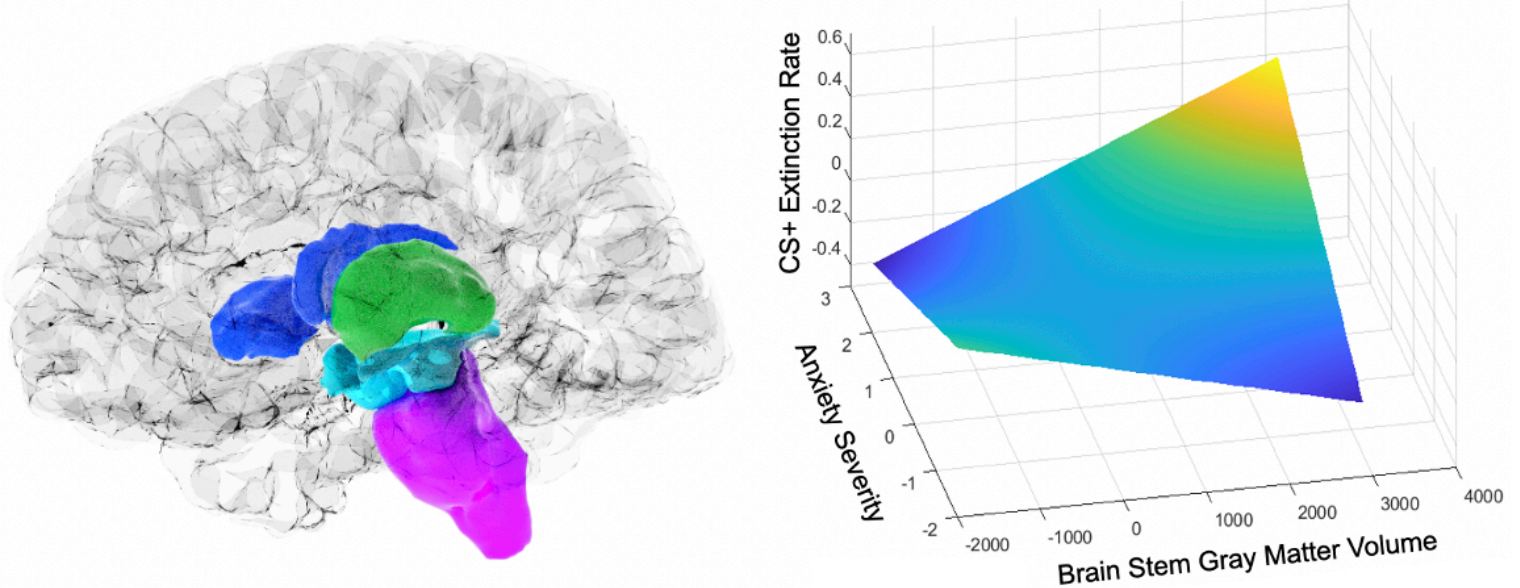

Figure 4. A) Empirical data for skin conductance response to CS+ across extinction trials (full lines; bars reflect one standard error of the mean) and fitted data (dashed line) by the model. B) Scatterplot depicts correlation between anxiety severity and CS+ extinction rate. C) Left: Gray matter volume in several subcortical structures moderated the association between CS+ extinction learning rate and anxiety severity: brain stem (purple), bilateral ventral diencephalon (light blue), bilateral caudate nucleus (dark blue), 
and left putamen (green). Right: in these structures, a similar pattern emerged for the interaction which is depicted in a plane; representative data from the brain stem are depicted. Warmer and cooler colors reflect more positive and negative values, respectively. All brain structure effects are significant at $p_{\mathrm{FWER}}<0.05$. 


\section{Discussion}

This study modeled trial-by-trial psychophysiological data to quantify threat learning processes and their associations with anxiety symptoms, neuroanatomy, and age ${ }^{1-3,19}$. Several key findings emerged. First, threat conditioning was optimally modeled with a RW model that featured Bayesian (diminishing) threat-learning rate and a habituation rate; habituation rate was associated with thalamus GMV. Second, using a similar model to characterize response to safety cues during conditioning, threat generalization rate was found to relate to anxiety severity only in younger age, whereas greater anxiety predicted slower safety learning across the sample. Finally, extinction learning featured exponential decay of response to threat cues, with slower decay being associated with greater anxiety. Further, GMV of multiple subcortical structures moderated the association between extinction learning rate and anxiety, such that as GMV was smaller, slower threat extinction was associated with greater anxiety severity. This study identifies specific latent threat-learning parameters that relate to anxiety symptom severity and neuroanatomical features.

The optimal fit for threat conditioning was a RW model that assumes Bayesian learning decay and habituation terms. A RW learning rule has long been proposed to underlie reinforcement learning, whereby point estimates of future outcomes are calculated by modifying current predictions according to prediction error, as weighted by a fixed learning rate ${ }^{27}$. Models based on a RW rule have been shown to describe the acquisition of conditioned fear responses in animals and humans ${ }^{69}$. Here, we show that a decrementally-diminishing threat-learning rate provides a better fit to physiology data in humans, suggesting that conditioning of threatanticipatory physiological responses is governed by a set learning parameter that is weighted less in proportion to additional reinforcement trials. It therefore places greater emphasis on learning 
from initial encounters with danger, supporting an adaptive role for this learning process in environments that contain threats ${ }^{3}$.

The inclusion of a CS+ habituation term could indicate that conditioned responding decreases as this cue becomes more predictable, beyond what is explained by the basic RW model itself ${ }^{27}$. This could be a product of the $80 \%$ reinforcement schedule used here; a different reinforcement ratio could have affected this habituation term. Additionally, the habituation term could reflect our use of trial-level UCS value when modeling, rather than $0 / 1$ as is often done, such that the magnitude of response to the UCS contributes to learning, rather than just its absence/presence. Indeed, response to UCS in conditioning paradigms is often shown to naturally diminish as learning is achieved, potentially reflecting effective and reliable anticipation of $\mathrm{it}^{40,70,71}$. Further, in other work ${ }^{72}$ we show that increasing UCS potency results in increasing physiological response to the UCS. The response habituation term in the model may therefore account for individual differences in dynamic changes in the strength of response that both CS+ and UCS elicit. Our data suggest that this process involves thalamus GMV, such that greater GMV is associated with faster habituation. Indeed, thalamus has been shown to relay information on conditioned and unconditioned stimuli during threat conditioning ${ }^{73}$. Our findings suggest that thalamus structure may play a more specific role relating to CS+ habituation. Threat conditioning parameters, however, were not associated with anxiety, suggesting that variations in symptom severity are not related to variations in the generation of threat associations ${ }^{1,58}$.

Of note, prior work identified the RW-PH "hybrid" model as providing optimal fit to data for the expression of threat contingencies among healthy adults as well as adults with PTSD 12,30,31. In contrast, we found that this model did not provide the best fit for rapid threat conditioning among the models tested. This discrepancy could potentially be attributed to the 
nature of threat learning processes studied. While we focused on rapid acquisition of threat contingencies which takes place over a short training schedule, prior studies examined threat contingencies over much longer durations (over $80 \mathrm{CS}+$ trials). The hybrid model includes a cumulative associability term which could be more sensitive to tracking contingency values or the expression of conditioned fear as it is optimized over longer durations. Thus, differences in rapid, crude acquisition vs optimized expression of threat contingencies processes could account for model differences. As such, the current and prior work could be seen as complementary in terms of threat learning processes studied, and both sets of findings could usefully inform study design for future work.

This study extends prior work by modeling additional learning effects that were evident in the data during threat conditioning. These additional effects showed associations with anxiety, age, and neuroanatomy. The introduction of an aversive UCS led to generalization of conditioned fear response to the CS- occurring most strongly early in conditioning, and followed by safety learning as this generalized response was inhibited as the CS- was not reinforced. Accordingly, this pattern of conditioned response to the CS- was modeled using two learning parameters. Learning theories of anxiety attribute each of these processes a role in the emergence and maintenance of pathological anxiety ${ }^{1,2,55,57,59}$. Our modeling approach allowed us to test these theories, providing some support for them, as well as for neurodevelopmental theories of anxiety ${ }^{19,23}$. First, threat generalization rate was significantly associated with anxiety severity, but only in participants under the age of 13 years. Given that anxiety symptoms typically emerge in late childhood and early adolescence ${ }^{17,74}$, this could potentially relate to aberrant threat generalization. It should be noted, however, that generalization rate was found to negatively correlate with anxiety severity, in contrast to theory ${ }^{59}$. This finding could potentially suggest that 
generalization of conditioned responses may be normative and adaptive in children, whereas anxiety could involve limited generalization. Additional research in child samples could further clarify the role of this effect in anxiety. Second, our findings provide support for prior propositions ${ }^{1,75}$ that link greater anxiety with slower safety learning rates ${ }^{1,22}$. This suggests that fear responses to aversive events may initially generalize to neutral stimuli, but that continued responding to these stimuli may contribute to the persistent and generalized fears associated with anxiety. Importantly, the use of computational modeling enabled us to extend prior work by directly examining associations between learning rates and anxiety.

Finally, our results show that threat extinction learning follows an exponential decay pattern. As in threat generalization and safety learning (see above), this is the first study to quantify the temporal dynamics of this learning process via modeling. Further, we show that slower extinction learning is associated with greater anxiety severity, providing support for major theories of anxiety and its treatment $t^{1,25,15,22,56,57,76}$. Thus, individuals with higher anxiety symptoms continue to express conditioned fear responses although the CS+ no longer predicts danger. This effect involves a number of subcortical structures, including brainstem, diencephalon, and basal ganglia. Across these, a similar moderation pattern was noted, such that among individuals with smaller GMV, slower extinction was associated with greater anxiety severity. This finding converges with prior work in animals and humans that suggests the involvement of at least some of these structures in threat extinction, such as caudate, hypothalamus, and midbrain/brainstem ${ }^{37,77,78}$. These regions are hypothesized to contribute to encoding of prediction errors and to physiological and behavioral aspects of response to threat ${ }^{7,76,78}$. Our findings extend prior work by linking variation in GMV of these subcortical structures, extinction learning, and anxiety. It should be noted that functional imaging work in 
humans identifies extensive cortical involvement during threat extinction paradigms. Our findings suggest that extinction learning rates, which are not assessed in prior work, may depend on more evolutionary-conserved subcortical structures, although studies that integrate functional imaging and modeling of threat extinction learning are required to further support this hypothesis. At the same time, it should be acknowledged that administering threat conditioning and extinction paradigms during scanning to anxious individuals, particularly youth, is challenging due to the aversive nature of such paradigms ${ }^{72,79}$.

This study utilized reinforcement modeling to reveal novel links between specific threatlearning processes and anxiety symptom severity, and identify neuroanatomical moderators of these associations. The findings generated from this application of a computational approach to link biological, clinical, and imaging data could initiate continued research along several lines. In terms of clinical research, the identified associations between threat learning processes and anxiety severity could inform theories on the etiology of anxiety symptoms and guide studies that aim to further qualify the learning conditions and mechanisms that promote anxiety ${ }^{13,57}$. Further, influential theories that place threat extinction processes at the center of exposure-based therapy $y^{5,22,56,76}$ could be evaluated more sensitively using modeling-derived indices. Translational research on mechanisms of threat learning could also benefit from these findings. Thus, neuroscience research in humans could focus on the specific processes identified here and extend our structural imaging findings to insight on function within this circuitry. Research in animals could complement such work by further delineating, via invasive manipulations ${ }^{80}$, the roles in threat learning of the subcortical structures identified here.

Several important limitations should be acknowledged. First, modeling was based on relatively few task trials. While we focused on the specific processes of rapid learning of threat 
associations that take place over very few pairings ${ }^{3}$, this could have led to noisier parameter estimates that could diminish accuracy and statistical power. Second, this was a cross-sectional study; a longitudinal design examining whether learning rates predict later emergence of symptoms would allow stronger inferences about developmental processes ${ }^{81}$. Third, only structural MRI data were examined; functional imaging during threat learning could reveal additional correlates of learning circuitry ${ }^{12}$, although the delivery of aversive stimuli to participants with anxiety in the MRI scanner presents a challenge ${ }^{79}$. Several strengths mitigate these limitations and address general shortcomings in threat learning research ${ }^{8,25}$. First, the large sample size increases precision of estimated parameters ${ }^{82}$, offsetting, to some extent, the small number of trials used for modelling. Second, participants were carefully assessed and free of medications known to impact threat learning and psychophysiology ${ }^{8}$. Third, wide anxietysymptom and age ranges generate inferences with reasonable statistical power. Fourth, task and setting were identical for all participants, reducing measurement confounds and noise.

In conclusion, in this study we used computational modeling to index dynamic learning processes associated with threat learning. Through this modeling approach, we quantified these learning processes, revealed specific associations with anxiety symptoms and age, and identified neuroanatomical substrates. These findings extend our knowledge of how these learning processes manifest in humans and how variations in these could potentially contribute to anxiety symptomatology. 


\section{References}

1 Mineka, S. \& Oehlberg, K. The relevance of recent developments in classical conditioning to understanding the etiology and maintenance of anxiety disorders. Acta Psychologica (Amst) 127, 567-580, doi:10.1016/j.actpsy.2007.11.007 (2008).

2 Duits, P. et al. Updated meta-analysis of classical fear conditioning in the anxiety disorders. Depression and Anxiety 32, 239-253, doi:10.1002/da.22353 (2015).

3 Fanselow, M. S. The role of learning in threat imminence and defensive behaviors. Curr Opin Behav Sci 24, 44-49, doi:10.1016/j.cobeha.2018.03.003 (2018).

4 LeDoux, J. E. Coming to terms with fear. Proceedings of the National Academy of Sciences of the United States of America 111, 2871-2878, doi:10.1073/pnas.1400335111 (2014).

5 Milad, M. R. \& Quirk, G. J. Fear Extinction as a Model for Translational Neuroscience: Ten Years of Progress. Annual Review of Psychology 63, 129-151, doi:DOI 10.1146/annurev.psych.121208.131631 (2012).

6 LeDoux, J. E. Emotion circuits in the brain. Annual Review of Neuroscience 23, 155-184, doi:10.1146/annurev.neuro.23.1.155 (2000).

7 Adolphs, R. The Biology of Fear. Current Biology 23, R79-R93, doi:10.1016/j.cub.2012.11.055 (2013).

8 Lonsdorf, T. B. et al. Don't fear 'fear conditioning': Methodological considerations for the design and analysis of studies on human fear acquisition, extinction, and return of fear. Neuroscience and Biobehavioral Reviews 77, 247-285, doi:10.1016/j.neubiorev.2017.02.026 (2017). 
9 Maren, S. Neurobiology of Pavlovian fear conditioning. Annual Review of Neuroscience 24, 897-931, doi:10.1146/annurev.neuro.24.1.897 (2001).

10 Maren, S. \& Quirk, G. J. Neuronal signalling of fear memory. Nature Reviews Neuroscience 5, 844-852, doi:10.1038/nrn1535 (2004).

11 Garrison, J., Erdeniz, B. \& Done, J. Prediction error in reinforcement learning: a metaanalysis of neuroimaging studies. Neuroscience and Biobehavioral Reviews 37, 12971310, doi:10.1016/j.neubiorev.2013.03.023 (2013).

12 Homan, P. et al. Neural computations of threat in the aftermath of combat trauma. Nature Neuroscience 22, 470-476, doi:10.1038/s41593-018-0315-x (2019).

13 Li, S. S. Y. \& McNally, G. P. The conditions that promote fear learning: Prediction error and Pavlovian fear conditioning. Neurobiology of Learning and Memory 108, 14-21, doi:10.1016/j.nlm.2013.05.002 (2014).

14 Lissek, S. et al. Classical fear conditioning in the anxiety disorders: a meta-analysis. Behaviour Research and Therapy 43, 1391-1424, doi:S0005-7967(04)00251-7 [pii] 10.1016/j.brat.2004.10.007 (2005).

15 Barlow, D. H. Anxiety and Its Disorders: The Nature and Treatment of Anxiety and Panic. (The Guilford Press, 2002).

16 Corchs, F. \& Schiller, D. Threat-related disorders as persistent motivational states of defense. Curr Opin Behav Sci 26, 62-68, doi:10.1016/j.cobeha.2018.10.007 (2019).

17 Beesdo, K., Knappe, S. \& Pine, D. S. Anxiety and anxiety disorders in children and adolescents: developmental issues and implications for DSM-V. Psychiatric Clinics of North America 32, 483-524, doi:10.1016/j.psc.2009.06.002 (2009). 
18 Kessler, R. C. et al. Lifetime co-morbidity of DSM-IV disorders in the US National Comorbidity Survey Replication Adolescent Supplement (NCS-A). Psychological Medicine 42, 1997-2010, doi:10.1017/S0033291712000025 (2012).

19 Casey, B. J., Glatt, C. E. \& Lee, F. S. Treating the Developing versus Developed Brain: Translating Preclinical Mouse and Human Studies. Neuron 86, 1358-1368, doi:10.1016/j.neuron.2015.05.020 (2015).

20 Pattwell, S. S. et al. Altered fear learning across development in both mouse and human. Proceedings of the National Academy of Sciences of the United States of America 109, 16318-16323, doi:10.1073/pnas.1206834109 (2012).

21 Lau, J. Y. et al. Distinct neural signatures of threat learning in adolescents and adults. Proceedings of the National Academy of Sciences of the United States of America 108, 4500-4505, doi:10.1073/pnas.10054941081005494108 [pii] (2011).

22 Craske, M. G., Hermans, D. \& Vervliet, B. State-of-the-art and future directions for extinction as a translational model for fear and anxiety. Philos $T R$ Soc B 373, doi:10.1098/rstb.2017.0025 (2018).

23 Craske, M. G. et al. Elevated responding to safe conditions as a specific risk factor for anxiety versus depressive disorders: evidence from a longitudinal investigation. Journal of Abnormal Psychology 121, 315-324, doi:10.1037/a0025738 (2012).

24 Dvir, M., Horovitz, O., Aderka, I. M. \& Shechner, T. Fear conditioning and extinction in anxious and non-anxious youth: A meta-analysis. Behaviour Research and Therapy, doi:https://doi.org/10.1016/j.brat.2019.103431 (2019).

25 Ney, L. J. et al. Critical evaluation of current data analysis strategies for psychophysiological measures of fear conditioning and extinction in humans. 
International Journal of Psychophysiology 134, 95-107,

doi:10.1016/j.ijpsycho.2018.10.010 (2018).

26 Keiflin, R. \& Janak, P. H. Dopamine Prediction Errors in Reward Learning and Addiction: From Theory to Neural Circuitry. Neuron 88, 247-263, doi:10.1016/j.neuron.2015.08.037 (2015).

27 Rescorla, R. A. \& Wagner, A. R. in Classical Conditioning II (ed A. H. Black \& W. F. Prokasy) 64-99 (Appleton-Century-Crofts, New York, 1972).

28 Schultz, W. Updating dopamine reward signals. Current Opinion in Neurobiology 23, 229-238, doi:10.1016/j.conb.2012.11.012 (2013).

29 Lee, D., Seo, H. \& Jung, M. W. Neural basis of reinforcement learning and decision making. Annual Review of Neuroscience 35, 287-308, doi:10.1146/annurev-neuro062111-150512 (2012).

30 Tzovara, A., Korn, C. W. \& Bach, D. R. Human Pavlovian fear conditioning conforms to probabilistic learning. PLOS Computational Biology 14, e1006243, doi:10.1371/journal.pcbi.1006243 (2018).

31 Zhang, S. Y., Mano, H., Ganesh, G., Robbins, T. \& Seymour, B. Dissociable Learning Processes Underlie Human Pain Conditioning. Current Biology 26, 52-58, doi:10.1016/j.cub.2015.10.066 (2016).

32 Li, J., Schiller, D., Schoenbaum, G., Phelps, E. A. \& Daw, N. D. Differential roles of human striatum and amygdala in associative learning. Nature Neuroscience 14, 12501252, doi:10.1038/nn.2904 (2011). 
33 Schiller, D., Levy, I., Niv, Y., LeDoux, J. E. \& Phelps, E. A. From fear to safety and back: reversal of fear in the human brain. Journal of Neuroscience 28, 11517-11525, doi:10.1523/JNEUROSCI.2265-08.2008 (2008).

34 Abend, R. et al. Anticipatory Threat Responding: Relationships with Anxiety, Development, and Brain Structure. Biological Psychiatry 87, 916-925 (2020).

35 Michalska, K. J. et al. Anxiety symptoms and children's eye gaze during fear learning. Journal of Child Psychology and Psychiatry, doi:10.1111/jcpp.12749 (2017).

36 Curzon, P., Rustay, N. R. \& Browman, K. E. in Methods of Behavior Analysis in Neuroscience (ed J. J. Buccafusco) (CRC Press/Taylor \& Francis, 2009).

37 Fullana, M. A. et al. Fear extinction in the human brain: A meta-analysis of fMRI studies in healthy participants. Neuroscience and Biobehavioral Reviews 88, 16-25, doi:10.1016/j.neubiorev.2018.03.002 (2018).

38 Fullana, M. A. et al. Neural signatures of human fear conditioning: an updated and extended meta-analysis of fMRI studies. Molecular Psychiatry 21, 500-508, doi:10.1038/mp.2015.88 (2016).

39 Shechner, T. et al. Fear conditioning and extinction in anxious and nonanxious youth and adults: examining a novel developmentally appropriate fear-conditioning task. Depression and Anxiety 32, 277-288, doi:10.1002/da.22318 (2015).

40 Britton, J. C. et al. Response to Learned Threat: An fMRI Study in Adolescent and Adult Anxiety. American Journal of Psychiatry 170, 1195-1204, doi:DOI

10.1176/appi.ajp.2013.12050651 (2013). 
41 Gold, A. L. et al. Age Differences in the Neural Correlates of Anxiety Disorders: An fMRI Study of Response to Learned Threat. American Journal of Psychiatry 177, 454463, doi:10.1176/appi.ajp.2019.19060650 (2020).

42 Birmaher, B. et al. The screen for child anxiety related emotional disorders (SCARED): Scale construction and psychometric characteristics. J Am Acad Child Psy 36, 545-553, doi:Doi 10.1097/00004583-199704000-00018 (1997).

43 Spielberger, C. D., Gorsuch, R. L. \& Lushene, R. E. Manual for the State-Trait Anxiety Inventory. (Consulting Psychologists Press, 1970).

44 Birmaher, B. et al. Psychometric properties of the Screen for Child Anxiety Related Emotional Disorders (SCARED): A replication study. J Am Acad Child Psy 38, 12301236, doi:Doi 10.1097/00004583-199910000-00011 (1999).

45 Behrens, B., Swetlitz, C., Pine, D. S. \& Pagliaccio, D. The Screen for Child Anxiety Related Emotional Disorders (SCARED): Informant Discrepancy, Measurement Invariance, and Test-Retest Reliability. Child Psychiatry \& Human Development, doi:10.1007/s10578-018-0854-0 (2018).

46 Abend, R. et al. A Computational Network Perspective on Pediatric Anxiety. Psychological Medicine (2020).

47 Elwood, L. S., Wolitzky-Taylor, K. \& Olatunji, B. O. Measurement of anxious traits: a contemporary review and synthesis. Anxiety Stress Copin 25, 647-666, doi:Doi 10.1080/10615806.2011.582949 (2012).

48 Abend, R. et al. Age Moderates Link Between Training Effects and Treatment Response to Attention Bias Modification Treatment for Social Anxiety Disorder. Journal of Abnormal Child Psychology 47, 881-894, doi:10.1007/s10802-018-0494-7 (2019). 
49 Den, M. L., Graham, B. M., Newall, C. \& Richardson, R. Teens that fear screams: A comparison of fear conditioning, extinction, and reinstatement in adolescents and adults. Dev Psychobiol 57, 818-832, doi:10.1002/dev.21330 (2015).

50 Lau, J. Y. et al. Fear conditioning in adolescents with anxiety disorders: results from a novel experimental paradigm. Journal of the American Academy of Child \& Adolescent Psychiatry 47, 94-102, doi:10.1097/chi.0b01e31815a5f01 S0890-8567(09)62089-X [pii] (2008).

51 Ryan, K. M., Zimmer-Gembeck, M. J., Neumann, D. L. \& Waters, A. M. The need for standards in the design of differential fear conditioning and extinction experiments in youth: A systematic review and recommendations for research on anxiety. Behaviour Research and Therapy 112, 42-62, doi:10.1016/j.brat.2018.11.009 (2019).

52 Tottenham, N. et al. The NimStim set of facial expressions: judgments from untrained research participants. Psychiatry Research 168, 242-249, doi:S0165-1781(08)00148-0 [pii] 10.1016/j.psychres.2008.05.006 (2009).

53 Marin, M. F. et al. Skin Conductance Responses and Neural Activations During Fear Conditioning and Extinction Recall Across Anxiety Disorders. JAMA Psychiatry 74, 622631, doi:10.1001/jamapsychiatry.2017.0329 (2017).

54 Marin, M. F. et al. Absence of conditioned responding in humans: A bad measure or individual differences? Psychophysiology, e13350, doi:10.1111/psyp.13350 (2019).

55 Dymond, S., Dunsmoor, J. E., Vervliet, B., Roche, B. \& Hermans, D. Fear Generalization in Humans: Systematic Review and Implications for Anxiety Disorder Research. Behavior Therapy 46, 561-582, doi:10.1016/j.beth.2014.10.001 S0005-7894(14)00134-8 [pii] (2015). 
56 Pittig, A., van den Berg, L. \& Vervliet, B. The key role of extinction learning in anxiety disorders: behavioral strategies to enhance exposure-based treatments. Current Opinion in Psychiatry 29, 39-47, doi:10.1097/Yco.0000000000000220 (2016).

57 Vervliet, B., Baeyens, F., Van den Bergh, O. \& Hermans, D. Extinction, generalization, and return of fear: A critical review of renewal research in humans. Biological Psychology 92, 51-58, doi:10.1016/j.biopsycho.2012.01.006 (2013).

58 Orr, S. P. et al. De novo conditioning in trauma-exposed individuals with and without posttraumatic stress disorder. Journal of Abnormal Psychology 109, 290-298, doi:Doi 10.1037/0021-843x.109.2.290 (2000).

59 Lissek, S. et al. Generalized Anxiety Disorder Is Associated With Overgeneralization of Classically Conditioned Fear. Biological Psychiatry 75, 909-915, doi:10.1016/j.biopsych.2013.07.025 (2014).

60 Abbott, A. Psychiatric genetics: The brains of the family. Nature 454, 154-157, doi:454154a [pii] 10.1038/454154a (2008).

61 Bishop, C. M. Neural Networks for Pattern Recognition. (Clarendon Press, 1995).

62 Schwarz, G. E. Estimating the dimension of a model. Annals of Statistics 6, 461-464 (1978).

63 Gold, A. L. et al. Cortical Thickness and Subcortical Gray Matter Volume in Pediatric Anxiety Disorders. Neuropsychopharmacology 42, 2423-2433, doi:10.1038/npp.2017.83 (2017).

64 Gold, A. L. et al. Comparing Brain Morphometry Across Multiple Childhood Psychiatric Disorders. J Am Acad Child Psy 55, 1027-1037 e1023, doi:10.1016/j.jaac.2016.08.008 (2016). 
65 Winkler, A. M., Ridgway, G. R., Webster, M. A., Smith, S. M. \& Nichols, T. E. Permutation inference for the general linear model. Neuroimage 92, 381-397, doi:10.1016/j.neuroimage.2014.01.060 (2014).

66 Smith, S. M. \& Nichols, T. E. Threshold-free cluster enhancement: addressing problems of smoothing, threshold dependence and localisation in cluster inference. Neuroimage 44, 83-98, doi:10.1016/j.neuroimage.2008.03.061 (2009).

67 Nordenskjold, R. et al. Intracranial volume normalization methods: Considerations when investigating gender differences in regional brain volume. Psychiat Res-Neuroim 231, 227-235, doi:10.1016/j.psychresns.2014.11.011 (2015).

68 Ruigrok, A. N. V. et al. A meta-analysis of sex differences in human brain structure. Neuroscience and Biobehavioral Reviews 39, 34-50, doi:10.1016/j.neubiorev.2013.12.004 (2014).

69 Herry, C. \& Johansen, J. P. Encoding of fear learning and memory in distributed neuronal circuits. Nature Neuroscience 17, 1644-1654, doi:10.1038/nn.3869 (2014).

70 Goodman, A. M., Harnett, N. G. \& Knight, D. C. Pavlovian conditioned diminution of the neurobehavioral response to threat. Neuroscience and Biobehavioral Reviews 84, 218-224, doi:10.1016/j.neubiorev.2017.11.021 (2018).

71 Johansen, J. P., Tarpley, J. W., LeDoux, J. E. \& Blair, H. T. Neural substrates for expectation-modulated fear learning in the amygdala and periaqueductal gray. Nature Neuroscience 13, 979-U102, doi:10.1038/nn.2594 (2010).

72 Abend, R. et al. Threat-anticipatory psychophysiological response is enhanced in pediatric anxiety and correlates with prefrontal cortex structure. Journal of Psychiary and Neuroscience (In press). 
73 Lanuza, E., Nader, K. \& Ledoux, J. E. Unconditioned stimulus pathways to the amygdala: Effects of posterior thalamic and cortical lesions on fear conditioning. Neuroscience 125, 305-315, doi:10.1016/j.neuroscience.2003.12.034 (2004).

74 Kessler, R. C. et al. Lifetime prevalence and age-of-onset distributions of DSM-IV disorders in the National Comorbidity Survey Replication. Archives of General Psychiatry 62, 593-602, doi:10.1001/archpsyc.62.6.593 (2005).

75 Lissek, S. et al. Impaired discriminative fear-conditioning resulting from elevated fear responding to learned safety cues among individuals with panic disorder. Behaviour Research and Therapy 47, 111-118, doi:10.1016/j.brat.2008.10.017 (2009).

76 Papalini, S., Beckers, T. \& Vervliet, B. Dopamine: from prediction error to psychotherapy. Translational Psychiatry 10, doi:10.1038/s41398-020-0814-x (2020).

77 Herry, C. et al. Neuronal circuits of fear extinction. European Journal of Neuroscience 31, 599-612, doi:10.1111/j.1460-9568.2010.07101.x (2010).

78 Stockhorst, U. \& Antov, M. I. Modulation of Fear Extinction by Stress, Stress Hormones and Estradiol: A Review. Frontiers in Behavioral Neuroscience 9, doi:10.3389/fnbeh.2015.00359 (2016).

79 Thorpe, S., Salkovskis, P. M. \& Dittner, A. Claustrophobia in MRI: the role of cognitions. Magnetic Resonance Imaging 26, 1081-1088, doi:10.1016/j.mri.2008.01.022 S0730-725X(08)00043-X [pii] (2008).

80 Likhtik, E. \& Paz, R. Amygdala-prefrontal interactions in (mal)adaptive learning. Trends in Neurosciences 38, 158-166, doi:10.1016/j.tins.2014.12.007 (2015).

81 Lonsdorf, T. B. \& Merz, C. J. More than just noise: Inter-individual differences in fear acquisition, extinction and return of fear in humans - Biological, experiential, 
temperamental factors, and methodological pitfalls. Neuroscience and Biobehavioral

Reviews 80, 703-728, doi:10.1016/j.neubiorev.2017.07.007 (2017).

82 Asendorpf, J. B. et al. Recommendations for Increasing Replicability in Psychology. Eur J Personality 27, 108-119, doi:10.1002/per.1919 (2013). 


\section{Acknowledgements}

We thank the participants and families, as well as the staff of the Intramural Research

Program of the National Institute of Mental Health (IRP, NIMH), National Institutes of Health.

We also thank Emily Ronkin, Elizabeth Steuber, Madeline Farber, Jessica Sachs, Brigid

Behrens, Carolyn Spiro, and Omri Lily for their contribution to data collection.

This research was supported (in part) by the NIMH IRP (ZIAMH002781-15,

NCT00018057; DSP) and NIH grant K99/R00MH091183 (JCB). 